\title{
Les résistances à la vaccination
}

> La vaccination est l'objet de controverses malgré son efficacité et bien que la très grande majorité de la population et des professionnels de santé y soit favorable. II existe en fait des résistances sélectives vis-à-vis de vaccinations contre l'hépatite B, la grippe, le BCG, la coqueluche et la rougeole, qui ont fait l'objet de controverses. Ces dernières, nées avec la vaccination, peuvent avoir diverses origines: effets secondaires réels, plus souvent coïncidence temporelle entre la vaccination et le déclenchement d'une maladie qui apparaît habituellement à l'âge où I'on vaccine. Cette coïncidence peut être anticipée et sa gestion doit être améliorée par une optimisation des systèmes de pharmacovigilance et de la communication à ce propos. Les controverses peuvent aussi être déclenchées par des études scientifiques aisément publiées même lorsqu'elles sont entachées de biais, ou parfois par l'interprétation tendancieuse des interventions des autorités de santé, ou encore par les décisions de justice, difficilement compréhensibles pour le public. Tous ces éléments jettent le doute dans les esprits et ne font que conforter les opposants, d'autant que l'exclusion d'un risque est difficilement démontrable sur le plan scientifique. L'expérience montre qu'il est difficile de sortir d'une polémique. L'amélioration de la communication, à la fois vis-à-vis des professionnels et du grand public est essentielle : elle passe par l'utilisation adéquate des médias mais aussi par l'intervention des professionnels de terrain vaccinateurs, à condition qu'ils soient compétents, convaincus et motivés. >

La vaccination a représenté une avancée technologique considérable, que résume la citation de Stanley Plotkin : "The impact of vaccination on the health of the world's people is hard to exagerate. With the exception of safe

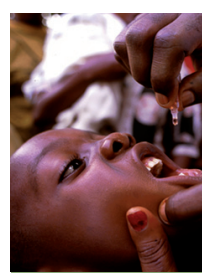

Président du Comité technique des vaccinations, Université Claude Bernard Lyon 1, Hôpital Femme-mère-enfant, 59 , boulevard Pinel, 69500 Bron, France. daniel.floret@chu-lyon.fr

water, no other modality, not even antibiotics, has had such a major effect on mortality reduction and population growth » [1]. Pourtant, la vaccination est, parmi les techniques médicales, celle qui a suscité le plus de controverses. Elle est également à l'origine de comportements paradoxaux : contraste entre une adhésion très satisfaisante lors d'enquêtes et les difficultés à obtenir des couvertures vaccinales adaptées. Paradoxe d'une demande très forte en cas de menace infectieuse («pourquoi n'y a-t-il pas de vaccin contre cette maladie?»), alors que l'acceptabilité est médiocre lorsque ce vaccin existe. Paradoxe entre une demande interventionniste (devant la recrudescence de rougeoles, «pourquoi ce vaccin n'est-il pas obligatoire? ») et les allégations d'atteinte aux libertés individuelles.

Bref, l'adhésion et la résistance aux vaccinations sont liées à des phénomènes complexes et multiformes et les problèmes qu'elles posent ne seront jamais aisés à résoudre.

\section{État actuel de l'opinion vis-à-vis de la vaccination en France}

Le grand public est globalement très en faveur de la vaccination (entre $90 \%$ et $95 \%$ ) bien que l'adhésion diminue avec l'âge et soit meilleure chez les hommes que chez les femmes [2].

Toutefois, $5 \%$ à $10 \%$ de la population exprime une opinion non favorable ou réservée vis-à-vis de la vaccination. Ceux-ci peuvent être classés en deux catégories [3].

1. Les sceptiques: souvent adeptes des médecines alternatives, ils sont en faveur de vaccinations sélectives et souhaitent des discussions sur les stratégies vaccinales, l'efficacité des vaccins, leur sécurité, les effets secondaires. 
2. Les opposants : ils représentent un groupe minoritaire et hétérogène avançant des motifs religieux, philosophiques ou idéologiques. Leurs arguments se classent dans plusieurs rubriques [4, 5] : violation des libertés individuelles, théorie du complot (les autorités de santé et les experts ont été achetés par l'industrie). Pour eux, les effets secondaires des vaccins sont minorés ou cachés. Les vaccins sont inefficaces, procurent une immunité artificielle et altèrent le système immunitaire. Ils prétendent que les vaccins ne sont pas responsables de la baisse d'incidence des maladies qui s'explique par l'amélioration des conditions de vie. En outre, ces vaccins sont dirigés contre les maladies communes de l'enfance, le plus souvent bénignes.

Du côté des professionnels de santé, une enquête de I'INPES (Institut national de prévention et d'éducation pour la santé) réalisée en 2009 [6] montre que $97,5 \%$ des médecins généralistes sont favorables aux vaccinations. Les médecins défavorables représentent $2 \%$ des médecins interrogés : ils pratiquent le plus souvent un exercice particulier, notamment l'homéopathie. Ils sont moins souvent installés en secteur 1 et font plus rarement partie d'un réseau de soins.

En somme, l'opinion tant du grand public que des professionnels de santé apparaît très favorable aux vaccinations, ce qui contraste avec les difficultés à obtenir des couvertures vaccinales adaptées. Cette adhésion globale cache des réticences sélectives vis-à-vis de certaines vaccinations, essentiellement celles contre l'hépatite $B$, la grippe, la rougeole, la coqueluche, le BCG qui, toutes, ont fait l'objet de controverses.

\section{Comment naissent les controverses sur la vaccination?}

Les controverses sur la vaccination sont nées avec la vaccination ellemême. Voltaire en 1734 [7] fait état de polémiques entre Européens à propos de la prévention de la variole par l'ancêtre de la vaccination (voir Encadré).

\section{Les faits réels}

Les controverses sont parfois nées des effets adverses réels liés aux vaccins. Ainsi, la vaccination antivariolique a causé des effets secondaires graves: encéphalites entraînant des décès et des séquelles neurologiques. Le vaccin vivant antipoliomyélitique a été à l'origine de cas de poliomyélite paralytique et même d'épidémies par la réacquisition d'une neurovirulence de la souche vaccinale dans un contexte de couverture vaccinale insuffisante [8].

En 1929-1930, à Lübeck, 72 enfants vaccinés par le BCG sont morts de tuberculose du fait d'une contamination de laboratoire lors de la préparation du vaccin BCG par une souche virulente de bacille de Koch.

\section{La relation temporelle}

Bien souvent, les polémiques ont pour origine la relation temporelle entre une vaccination et des effets secondaires graves, fait inévitable lorsqu'on vaccine les nourrissons au pic d'incidence de la survenue du syndrome de mort subite ${ }^{1}$ ou de la révélation de maladies métaboliques

${ }^{1}$ Le syndrome de la mort subite du nourrisson est défini comme le décès soudain d'un jeune enfant, inattendu au regard de son histoire, demeurant inexpliqué malgré les examens réalisés après la mort.

\section{Voltaire. Lettre XI,} Lettres philosophiques (1734) [7]

On dit doucement, dans l'Europe chrétienne, que les Anglais sont des fous et des enragés: des fous, parce qu'ils donnent la petite vérole à leurs enfants, pour les empêcher de l'avoir; des enragés, parce qu'ils communiquent de gaieté de cœur à ces enfants une maladie certaine et affreuse, dans la vue de prévenir un mal incertain. Les Anglais, de leur côté, disent: «les autres Européens sont des lâches et des dénaturés: ils sont lâches, en ce qu'ils craignent de faire un peu de mal à leurs enfants; dénaturés, en ce qu'ils les exposent à mourir un jour de la petite vérole».

ou de maladies neurologiques d'étiologie inconnue (hypsarythmie, syndrome de Rett, autisme, etc.) ; il en est de même lorsque l'on vaccine les adolescents à l'âge où se révèlent les maladies auto-immunes.

Les polémiques concernant la vaccination contre l'hépatite $B$ en France sont le plus bel exemple de l'effet pervers d'un tel lien temporel. En 1995, les autorités sanitaires suivent les préconisations de l'OMS (Organisation mondiale de la santé) et recommandent de vacciner contre l'hépatite B les nourrissons, les adolescents et les adultes à risque. La communication officielle sur ces recommandations, relayée par une campagne publicitaire agressive des firmes, provoque un engouement pour cette vaccination. Ainsi, plus de 75 millions de doses de vaccin sont vendues en deux ans et plusieurs millions d'adultes, non ciblés par les recommandations, se sont fait vacciner à l'âge où débute la sclérose en plaques (SEP). Il en résulte des notifications à l'Agence du médicament d'épisodes démyélinisants chez des sujets récemment vaccinés: ce fut le point de départ des polémiques sur la responsabilité de cette vaccination dans le déclenchement de la sclérose en plaques.

De même, le vaccin anticoquelucheux a été accusé en France d'être responsable de la mort subite chez des nourrissons, accusation réfutée par une enquête épidémiologique [9]. L'implication de ce vaccin dans la responsabilité de cas d'épilepsie grave, d'encéphalites et de séquelles neurologiques définitives a entraîné l'arrêt de la vaccination dans certains pays (Angleterre, Suède, Japon, etc.) avant que deux études épidémiologiques réalisées en Angleterre et aux États-Unis n'innocentent le vaccin de la responsabilité de ces complications graves $[10,11]$.

\section{Les études scientifiques}

Les polémiques sont volontiers déclenchées par des études dont la méthodologie est discutable et qui, 
curieusement, sont acceptées pour publication dans des revues scientifiques de bon niveau. De fait, publier sur le lien entre une maladie grave et une vaccination représente un gage de notoriété pour l'auteur et une excellente publicité pour la revue.

- Ainsi la controverse anglaise sur la responsabilité de la vaccination contre rougeole, rubéole et oreillons (dit vaccin ROR) dans la genèse de l'autisme fut déclenchée par une publication [12] décrivant chez 12 enfants une pathologie digestive associée à un tableau autistique. Les parents de 8 de ces enfants associent le début de ces troubles à la vaccination par le vaccin ROR. Des publications ultérieures tentent d'élucider le mécanisme physiopathologique de cette association [13]. - La myofasciite à macrophages ${ }^{2}$ est un autre exemple. Le syndrome de fatigue chronique est une affection dont la définition est peu précise et la réalité discutée. La découverte - lors de l'examen de biopsies musculaires de certains patients - de granulomes à corps étranger contenant de l'aluminium (adjuvant de l'immunité utilisé dans la plupart des vaccins tués) conduit à attribuer à l'aluminium présent dans les vaccins la responsabilité d'une entité proche du syndrome de fatigue chronique [14].

L'analyse montre que les doses d'aluminium administrées dans le cadre des vaccinations sont négligeables par rapport à l'apport alimentaire normal et que la présence d'un granulome contenant de l'aluminium au lieu d'injection des vaccins est un phénomène normal et connu (il est d'ailleurs déconseillé de pratiquer les biopsies musculaires dans le deltoïde, lieu habituel d'injection des vaccins). Enfin, il n'existe aucune corrélation entre la constatation d'un granulome au point d'injection des vaccins et une maladie systémique [15].

- L'analyse des derniers rebondissements de la polémique de la vaccination contre l'hépatite $B$ en France est également très instructive. Une équipe française a mis en place un suivi de cohorte d'enfants présentant une sclérose en plaques et a publié deux études démontrant que la vaccination contre l'hépatite $B$ des enfants ayant déjà présenté une poussée de SEP n'entraînait pas de rechute [16] puis que les enfants vaccinés contre l'hépatite $B$ ne présentaient pas de risque accru d'apparition ultérieure de SEP [17].

À partir de cette cohorte reprise et complétée, une troisième étude est réalisée qui montre à nouveau que la vaccination contre l'hépatite $B$ n'est pas associée à un risque accru de survenue d'un épisode de démyélinisation, quels que soient la marque du vaccin, le nombre de doses et le délai entre vaccination et le premier signe neurologique [18].

Les auteurs réalisent ensuite 160 tests dans des sous-groupes, ce qui permet l'identification a posteriori dans un sous-groupe d'enfants ayant une bonne compliance à la vaccination d'un test statistiquement significatif entre la survenue d'une poussée de démyélinisation et de SEP et la vaccination plus de trois ans auparavant avec le vaccin Engerix $B^{\circledR}$. Mais une telle analyse des données est méthodologiquement contestable dès lors qu'une absence de relation statistiquement significative est démontrée sur le critère de jugement principal.

${ }^{2}$ Tableau de myopathie inflammatoire observé depuis 1993 : il associe chez l'adulte des myalgies d'intensité variable, des douleurs articulaires, associées à une fatigue chronique invalidante. La biopsie musculaire révèle un infiltrat macrophagique.

\section{Les autorités de santé}

Certaines polémiques ont été déclenchées par des réactions non attendues aux interventions des autorités de santé.

Le thiomersal - un composé renfermant du mercure est ajouté aux vaccins depuis 1930 comme conservateur et antiseptique sans qu'aucun effet adverse n'ait été rapporté en relation avec cet additif. En 1999, aux États-Unis, la Federal drug administration (FDA) s'inquiète des quantités de mercure reçues par les nourrissons du fait des vaccins et met en place des études qui concluent que les doses de mercure administrées sont sans commune mesure avec les quantités ingérées par l'alimentation et qu'il n'y a pas de risque d'accumulation. Elle demande néanmoins aux producteurs de vaccins de retirer le thiomersal des vaccins monodose, cette adjonction n'étant aucunement nécessaire. Ces différentes interventions de la FDA et la publicité qui en est faite seront à l'origine de la polémique sur la responsabilité du thiomersal dans le déclenchement de l'autisme infantile [19].

\section{Quels sont les facteurs qui entretiennent les controverses?}

\section{Les mesures contraignantes}

La polémique relative à la vaccination antivariolique en Angleterre [20] est très instructive à cet égard: pour faire face à la variole l'Angleterre met en place à partir de 1808 des mesures destinées à assurer une vaccination gratuite, notamment des plus pauvres. La mise en échec des mesures successives aboutit finalement à la publication en 1853 du Vaccination act qui crée l'obligation vaccinale pour les enfants. Devant les difficultés d'application (absence de financement des vaccinateurs, tiédeur des autorités locales), un système contraignant se met progressivement en place afin d'obliger les parents à faire vacciner leurs enfants. Le résultat sera la création en 1866 de la première ligue antivaccinale puis le déclenchement d'une véritable guerre de tranchées: dénonciation de la dangerosité du vaccin (réelle !), de la privation de libertés. Les politiques s'élèvent contre le coût du système (déjà...) et des méthodes d'échappement s'organisent (faux noms, fausses adresses, soustraction par aspiration du produit vaccinal). Une véritable politique de désobéissance civile se met en place pour s'opposer à l'obligation vaccinale. En 1898, le Vaccination act introduit une clause de conscience permettant aux parents de devenir objecteurs, ce qui va finalement aboutir en 1907 à la suppression de fait de l'obligation vaccinale.

En France, la vaccination par le BCG a été rendue obligatoire en 1949. La non-application de cette mesure va 
entraîner en 1952 la mise en place d'un système contraignant allant jusqu'à prévoir des mesures d'emprisonnement pour les contrevenants et un contrôle en milieu scolaire. Peu après, en 1954, est créée la première ligue antivaccinale française.

Durant la récente campagne de vaccination contre la grippe A, l'organisation, qualifiée par certains de militaire, de la campagne de vaccination (centres de vaccination gérés par le ministère de l'Intérieur, réquisition des médecins vaccinateurs, etc.) est apparue comme un facteur de non-adhésion aux recommandations vaccinales.

\section{Une communication inadaptée} sur les effets adverses des vaccins

Les effets adverses listés dans le RCP (résumé des caractéristiques de produits) des vaccins donnent des cauchemars aux prescripteurs et aux patients. Ainsi, la SEP et le syndrome de Guillain-Barré ${ }^{3}$ figurent en tête dans la liste des effets secondaires neurologiques du vaccin contre l'hépatite $B$.

Par souci de transparence, un système spécifique de pharmacovigilance relatif aux notifications des effets secondaires de la vaccination contre l'hépatite $B$ a été mis en place en 1994, complété à partir de 1998 par les notifications, même rétrospectives, de l'association luttant pour la reconnaissance et l'indemnisation des effets adverses de la vaccination contre l'hépatite B (association REVAHB). Ainsi, depuis la mise en place de la vaccination jusqu'en 2006, 1364 cas de démyélinisations centrales ( 1139 SEP) et 111 cas de démyélinisation périphérique ont été notifiés. La traduction de ces données, livrées brutes et sans analyse de causalité par les médias (et opposants), est que la vaccination a causé 1139 cas de SEP en France ${ }^{4}$.

La communication au début de la campagne de vaccination contre la grippe A sur un cas de syndrome de Guillain-Barré (qui n'en était pas un) et un cas de mort fœtale in utero ont certainement contribué à entretenir la méfiance vis-à-vis des vaccins pandémiques [35]. Les études réalisées tant en France [21] qu'aux États-Unis [22] et dans l'Union Européenne [23] ont en effet montré que les craintes vis-à-vis de la sécurité des vaccins et la non-perception de la gravité de la grippe avaient représenté les motivations principales pour ne pas se faire vacciner.

\section{La tiédeur des autorités de santé}

Le silence prolongé des autorités de santé lors de la polémique sur la vaccination contre l'hépatite $B$ a créé un sentiment d'abandon chez les professionnels de santé vaccinateurs et a même semé le doute sur la réalité des accidents chez certains d'entre eux.

\section{Des décisions de justice incomprises}

D'aucuns s'étonnent de décisions judiciaires non conformes aux données scientifiques. En fait, la justice, indépendante, analyse la causalité selon d'autres critères que ceux utilisés par les scientifiques. Ainsi,

${ }^{3}$ Syndrome de Guillain-Barré : polyradiculonévrite aiguë inflammatoire post-infectieuse. L'atteinte des nerfs périphériques se caractérise par une faiblesse, voire une paralysie progressive débutant au niveau des jambes et remontant parfois jusqu'à atteindre les muscles de la respiration. Dans la majorité des cas, les patients récupèrent en 6 à 12 mois.

${ }^{4}$ Le Monde, 25 septembre 2008 à plusieurs reprises, les firmes ont été reconnues responsables de cas de sclérose en plaques. Ces décisions (à l'exception de la dernière en juin 2009) ont toutes été cassées; mais ces décisions de cassation n'ont été accompagnées d'aucune médiatisation, à l'inverse des condamnations initiales. Le trouble est entretenu par la contradiction entre la non-reconnaissance de la responsabilité du vaccin (et des firmes) pour les sujets vaccinés en population générale et l'indemnisation par l'État des professionnels de santé ayant déclaré une SEP à la suite d'une vaccination obligatoire en milieu professionnel. Les initiés savent que le statut juridique des vaccins obligatoires est différent de celui des autres vaccins (la victime n'ayant pas à apporter la preuve du lien de causalité en cas d'effet secondaire) et que le Conseil d'ćtat a considéré que le fait de présenter les premiers signes de la maladie dans le mois suivant l'administration d'une dose de ce vaccin représentait une suspicion suffisamment forte pour permettre une indemnisation. Cette décision est en revanche totalement incompréhensible pour le commun des mortels.

\section{La difficulté scientifique à exclure le risque}

Au plan scientifique, il est beaucoup plus facile de démontrer un lien entre deux faits que de l'exclure, d'autant que des données divergentes apparaissent nécessairement et par le simple fait du hasard lors de la multiplication des études. Ainsi, dans l'exemple de l'association entre la vaccination contre l'hépatite $B$ et les maladies démyélinisantes, quatre études ne montrent pas d'augmentation du risque. Quatre études montrent une augmentation du risque qui n'est pas statistiquement significative, qui ne permet pas de dire si cette augmentation est réelle ou le fait du hasard. Enfin, l'étude de Hernan [24] montre une augmentation du risque. Communiquer sur les biais (réels) de cette étude expose à des critiques de partialité (on ne trouve des biais méthodologiques que dans les études qui vont dans le mauvais sens). De ce fait, la communication s'est concentrée sur la balance bénéfice/risque, ce qui a été interprété comme une reconnaissance du risque.

\section{Qui relaie les controverses?}

Les médias sont un relais puissant, surtout lorsqu'ils sont de mauvaise foi et s'appuient sur le sensationnel et le pathétique. Sur un plateau de télévision, une patiente en fauteuil roulant affirmant (on ne peut lui reprocher d'en être convaincue) la relation entre sa maladie et la vaccination a infiniment plus de poids que l'expert qui essaie d'expliquer des notions compliquées de statistique. 
Les ligues antivaccinales s'engouffrent dans la brèche, d'autant plus facilement qu'elles peuvent s'appuyer sur des avis d'experts, fussentils autoproclamés.

Internet a montré, à l'occasion de la grippe A, son fort pouvoir de nuisance, par sa capacité à relayer et amplifier des informations non validées, voire grossièrement erronées.

\section{Comment en sortir?}

\section{Il est difficile de sortir d'une polémique}

- Malgré les études, les démentis, les conférences de consensus et autres conférences citoyennes, la polémique concernant la vaccination contre l'hépatite $B$ se poursuit depuis plus de quinze ans en France.

- Une quinzaine d'études réalisées dans divers pays (Royaume-Uni, Danemark, Finlande, Japon, Canada, États-Unis, etc.) ont démontré l'absence de lien entre la vaccination par le ROR et l'autisme infantile. Des manquements aux règles d'éthique et de graves conflits d'intérêt concernant Andrew Wakefield (financé par une association d'avocats réclamant de l'État une indemnisation des victimes) ont été mis en évidence causant une rétractation des co-auteurs de l'article [25], l'embarras et les excuses du Lancet $[26,27]$ puis le retrait de l'article du sommaire du journal [28]. Ni ces faits, ni probablement l'ordonnance de mai 2010 du General medical council demandant que A. Wakefield soit rayé de la liste des médecins du Royaume-Uni ne suffiront à arrêter les polémiques sur la vaccination contre la rougeole.

- La présence de thiomersal dans les vaccins pandémiques multidoses a été un sujet majeur de polémiques lors de la campagne de vaccination pandémique, malgré les études démontrant l'absence d'association entre ce produit et l'autisme infantile [29], y compris après une exposition fœtale [30].

- Les pays qui ont arrêté de vacciner les enfants contre la coqueluche ont vu augmenter rapidement le nombre de cas et de morts, ce qui a permis la reprise de la vaccination en Angleterre. Dans d'autres pays (Suède, Japon) il a fallu attendre la mise à disposition du vaccin coquelucheux acellulaire, mieux toléré, pour relancer la vaccination [31].
L'analyse que fait Chen [32] de l'adhésion à la vaccination est d'ailleurs à méditer: l'introduction d'une vaccination entraîne une chute de l'incidence de la maladie mais également, en parallèle, une augmentation de l'incidence des effets secondaires, de sorte qu'après un certain temps, seuls les effets adverses des vaccins apparaissent alors que la maladie, devenue très rare, a été oubliée. II s'ensuit une perte de confiance en la vaccination, une chute de la couverture vaccinale et la survenue d'une épidémie qui va restaurer la confiance, permettre la reprise de la vaccination et en définitive l'élimination de la maladie (Figure 1).

\section{Comment faire mieux?}

La communication est décisive. Elle doit s'adresser aux professionnels de santé et au grand public.

En théorie, les stratégies de communication à destination du public et des professionnels de santé sont différentes. En fait, et l'expérience de la pandémie l'a bien montré, l'accès croissant du grand public à Internet et le très large relais par les médias des études publiées dans les journaux scientifiques font que pratiquement tout le monde a accès aux mêmes informations. Ainsi, la communication par l'intermédiaire des médias (à condition de les intéresser et de bien maîtriser l'outil) atteint aussi efficacement le grand public que les professionnels. Ces mêmes moyens doivent être utilisés pour lutter contre la désinformation, notamment sur Internet.

Ainsi, la communication sur la vaccination doit s'accompagner d'une communication sur la maladie qui s'appuie sur des données factuelles, objectives, dépassionnées, dans des termes peu différents selon que l'on s'adresse au grand public ou aux professionnels. Les principales questions sont: qui communique et comment sont choisis les messages. L'implication de professionnels n'appartenant pas aux structures qui

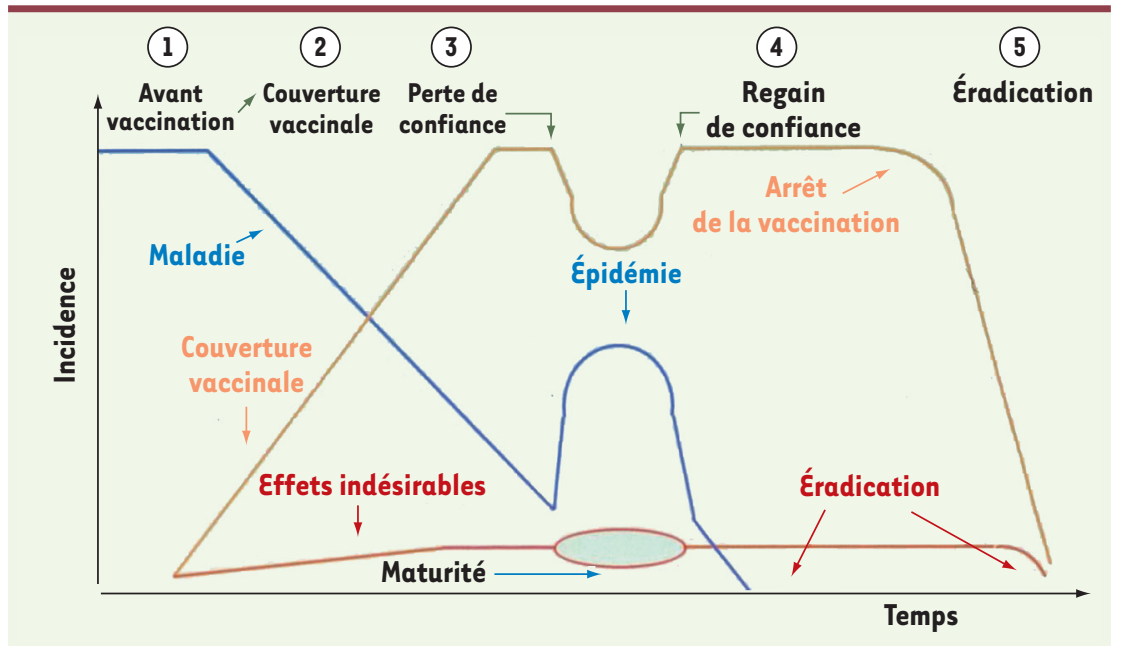

Figure 1. Évolution des programmes de vaccination (d'après [32]). 
élaborent les recommandations ou les procédures d'organisation de la vaccination (sociétés savantes) semble importante. Le choix des messages doit impliquer des professionnels d'autres horizons (sociologues, psychologues, anthropologues, etc.) et des membres de la société civile. En contrepartie, les autorités de santé devraient s'occuper des experts autoproclamés et communiquer sur leur légitimité.

Des leçons doivent être tirées des expériences passées: la pertinence de l'information et le moment que l'on choisit pour communiquer sur les effets adverses d'un vaccin relevant vraisemblablement d'une coïncidence doivent être mûrement réfléchis. Toute communication sur les effets indésirables notifiés devrait s'accompagner d'une analyse d'imputabilité, ce qui n'est pas simple. Concernant les effets adverses graves, il est fondamental de pouvoir évaluer rapidement le rapport cas observés/cas attendus. Ceci suppose une bonne connaissance de l'épidémiologie des maladies concernées et de prendre quelque recul par rapport à l'événement. L'amélioration du système de notification des effets indésirables permettant une meilleure exhaustivité en accroitrait la crédibilité.

On a vu l'impact possible des événements fortuits sur l'image de marque de la vaccination. Ceux-ci devraient être systématiquement anticipés lors de la mise en œuvre d'un nouveau programme de vaccination. Ainsi, une équipe de chercheurs a pu déterminer le nombre prévisible d'accidents allergiques, de crises d'asthme, de révélations de diabète ou de maladies auto-immunes à la suite d'une campagne de vaccination contre le papillomavirus [33]. À partir des bases de données sur les incidences de maladies ou évènements aux États-Unis et au Royaume-Uni, cette même équipe [34] a évalué le nombre de cas de syndrome de Guillain-Barré, de névrite optique, d'avortement et de mort subite qui devrait être notifié suite à une vaccination de masse contre la grippe pandémique $\mathrm{A}(\mathrm{HINl})$. Si la plupart des éléments de communication peuvent servir à la fois au grand public (via les médias) et aux professionnels (à travers la presse professionnelle mais aussi les médias), des spécificités doivent être considérées.

On sait qu'il existe vis-à-vis de la vaccination des opposants idéologiques. Ceux-ci ne seront pas convaincus. La stratégie vis-à-vis de ces personnes est de bien connaître leur argumentaire et d'être capable d'en démontrer les failles.

Les sceptiques, eux, sont susceptibles d'être convaincus, à condition d'avoir les bons arguments et de prendre le temps de la discussion. Les professionnels de terrain jouent en la matière un rôle essentiel. Leur nonadhésion à la vaccination contre le virus HINl pandémique a pesé lourd dans l'issue de la campagne vaccinale. Des efforts doivent être faits pour les motiver davantage, tout en notant que la compétence en vaccinologie nécessite un investissement lourd que tous les médecins ne sont pas prêts à effectuer.

\section{Conclusion}

La communication en santé publique est un art difficile. La résistance aux vaccins a toujours existé et semble inéluctable. II faut s'attendre à ce que tout nouveau programme de vaccination déclenche des polémiques et ce d'autant plus que des méthodes contraignantes voire coercitives auront été utilisées, surtout si le poids de la maladie n'est pas ressenti.
Toutefois, certaines polémiques peuvent et doivent être anticipées. On doit apprendre à mieux y faire face, notamment en se servant des mêmes armes que les opposants à la vaccination. Il convient par ailleurs d'éviter de les déclencher en créant la confusion au nom de la transparence. $\diamond$

\section{SUMMARY}

\section{Resistance to immunization}

Despite a well documented efficacy and a generally good adhesion of a large majority of the lay population and health care professionals as well, immunization is still the object of controversies. It affects several vaccines against hepatitis $B$, influenza, BCG, pertussis and measles. In most cases, polemics on vaccination result from a temporal association between a vaccination and the revelation of a serious disease, when the immunization is performed at the peak of incidence of the disease. Controversies can also be initiated by scientific publications, even though these are often biased, by a misinterpretation or the absence of official positions from the health care authorities, or by justice decisions, whose criteria differ from the scientists' ones and are therefore difficult to understand for the lay public. Furthermore, the scientific demonstration of the exclusion of a risk is difficult to obtain. In the past, it appeared to be very difficult to stop controversies. Improving the communication is the main issue, towards lay public as well as health care professionals. This implies to learn how to better use the medias, to motivate and educate professionals who administrate vaccine, and anticipate the eventuality of a temporal relationship between vaccination and occurrence of a serious disease by an improved management of the pharmacovogilance system. $\diamond$

\section{CONFLIT D'INTÉRÊTS}

Bien qu'ayant naguère déclaré des liens, de nature non financière, avec les firmes fabriquant des vaccins (réalisation d'études épidémiologiques, participation à des actions de formation, invitations à des congrès médicaux) et participé à des interventions ponctuelles (conférences, colloques, actions de formation) pour les entreprises Sanofi, Wyeth, GSK, l'auteur déclare n'avoir aucun conflit d'intérêts concernant les données publiées dans cet article.

\section{RÉFÉRENCES}

1. Plotkin SL, Plotkin SA. A short story of vaccination. In : Plotkin SA, Orenstein WA, eds. Vaccines. Philadelphia: WB Saunders, $2004: 1-15$.

2. INPES. Baromètre santé 2005. http://www.inpes.sante.fr/index2. asp?page=Barometres/Baro2005_1R/synthese/vaccination.asp

3. Jestin C. INPES. Communication personnelle.

4. Davies P, Chapman S, Leask J. Antivaccination activists on the world wide web. Arch Dis Child 2002; $87: 22-5$.

5. Wolfe RM, Sharp LK, Lipsky MS. Content and design attributes of antivaccination web sites. JAMA $2002 ; 287$ : 3245-8.

6. Jestin C, Gautier A. Perception de la vaccination par les médecins généralistes. Actualité et Dossier en Santé Publique 2010 ; 71 : 31 -4. 
7. Voltaire. Lettre $\mathrm{XI}$ : sur l'insertion de la petite vérole. In : Lettres philosophiques. 1734. http://www.voltaire-integral.com/Html/22/11_Lettre_1l.html

8. Kew OM, Wright PF, Agol VI, et al. Circulating vaccine-derived polioviruses: current state of knowledge. Bull World Health Organ $2004 ; 82$ : 16-23.

9. Messiah A, Flahaut $A$. Absence de liaison entre la vaccination tétravalente et le syndrome de mort subite du nourrisson (enquête cas-témoins DGS/Inserm 1986). BEH 1986; 52 : 205-6.

10. Miller DL, Ross EM, Alderslade R, et al. Pertussis immunisation and serious acute neurological illness in children. Br Med J $1981 ; 282$ : 1595-9.

11. Shields WD, Nielsen C, Buch D, et al. Relationship of pertussis immunization to the onset of neurologic disorders: a retrospective epidemiologic study. J Pediatr 1988; 113: 801-5.

12. Wakefield AJ, Murch SH, Anthony A, et al. Ileal lymphpoid nodular hyperplasia, non specific colitis, and pervasive developmental disorders in children. Lancet $1998 ; 351: 637-41$

13. Wakefield AJ. Enterocolitis, autism and measles virus. Mol Psychiatry 2002; 7 (suppl 2) : S44-6.

14. Gherardi RK, Coquet M, Chérin P, et al. Macrophagic myofasciitis: an emerging entity. Groupe d'étude et de recherche sur les maladies musculaires acquises et dysimmunitaires (GERMMAD) de I'Association française contre les myopathies (AFM). Lancet $1998 ; 352: 347-52$.

15. Siegrist CA. Les adjuvants vaccinaux et la myofasciite à macrophages. Arch Pediatr 2005 $12: 96-101$.

16. Mikaeloff $Y$, Caridade G, Assi S, et al. Hepatitis B vaccine and risk of relapse after a first childhood episode of CNS inflammatory demeylination. Brain 2007 ; 130 : 1105-10.

17. Mikaeloff $Y$, Caridade G, Rossier M, et al. Hepatitis B vaccination and the risk of childhood-onset multiple sclerosis. Arch Pediatr Adolesc Med 2007 ; 161 : 1176-82.

18. Mikaeloff Y, Caridade G, Suissa S, Tardieu M. Hepatitis B vaccine and the risk of CNS inflammatory demyelination in childhood. Neurology $2009 ; 72: 1260-6$.

19. Offit PA. Thimerosal and vaccines: a cautionary tale. N Engl J Med 2007; $357: 1278-9$.

20. Floret D. Faut-il abroger les obligations vaccinales ? Arch Pediatr $2006 ; 13: 423-5$

21. Schwarzinger M, Flicoteaux R, Cortarenoda $S$, et al. Low acceptability of $A / H 1 N 1$ pandemic vaccination in French adult population: did public health policy fuel public dissonance? PloS One $2010 ; 5$ : e10199.

22. SteelFisher GK, Blendon RJ, Bekheit MM, Lubell K. The public's response to the $2009 \mathrm{HINl}$ influenza pandemic. $N$ Engl J Med $2010 ; 362$ : e65

23. European Commission. Influenza H1N1. Analytical report. Publication : mars 2010. http:// ec.europa.eu/public_opinion/flash/fl_287_en.pdf.
24. Hernan MA, Jick SS, Olik MJ, Kick H. Recombinant hepatitis B vaccine and the risk of multiple sclerosis: a prospective study. Neurology $2004 ; 63: 638-42$

25. Murch SH, Anthony A, Casson DH, et al. Retraction of an interpretation. Lancet $2004 ; 363: 750$

26. Horton R. A statement by the editors of the Lancet. Lancet 2004 ; $363: 820-1$.

27. Lyall J. Editor in the eye of the storm. Br Med J $2004 ; 328: 528-8$.

28. The Editors of The Lancet. Retraction. Ileal-lymphoid hyperplasia, nonspecific colitis, and pervasive developmental disorder in children. Lancet $2010 ; 375: 445$.

29. Parker SK, Schwartz B, Todd J, Pickering LK. Thimerosal-containing vaccines and autistic spectrum disorder: a critical review of published original data Pediatrics $2004 ; 114$ : 793-804

30. Thompson WW, Price C, Goodson B, et al. Early thimerosal exposure and neurospychological outcomes at 7 and 10 years. $N$ Engl J Med 2007 357: 1281-92.

31. Kimura M. Japanese experience with acellular pertussis vaccines. Dev Biol Stand $1991 ; 73: 5-9$.

32. Chen RT, Orenstein WA. Epidemiologic methods in immunization programs. Epidemiol Rev 1996; 18 : 99-117.

33. Siegrist CA, Lewis $E M$, Eskola J, et al. Human papilloma virus immunization in adolescent and young adults. A cohort study to illustrate what events might be mistaken for adverse reactions. Pediatr Infect Dis J 2007 ; 26 : 979-84.

34. Black S, Eskola J, Siegrist CA, et al. Importance of background rates of disease in assessment of vaccine safety during mass immunisation with pandemic HINI influenza vaccines. Lancet $2009 ; 374: 2115-22$

35. Coignard-Biehler $\mathrm{H}$, Lortholary 0 . Cabale contre la vaccination anti-H1N1 est-elle justifiée? Med Sci (Paris) 2009; 25 : 967-70.
TIRÉS À PART

D. Floret

\section{L'ouvrage de référence, unique sur le marché francophone}

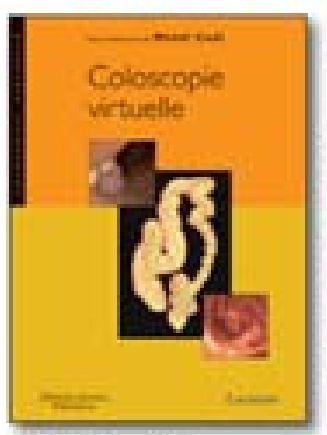

$24902010-49 \&$ 944 puger - 149 illitioncion couleurs et $\mathbf{N S B}=14$ tableme L65N $7.9782 .257 .00065 \cdot 2$

\section{Coloscopie virtuelle}

\section{Sous la direction de Mehdi CADI}

La coloscopie virtuelle, ou coloscanner, śest rapidement imposée comme une méthode nadiologique efficace dans la détection de polype et de cancer colorectal, et trouve sa place à obeć de la coloscopie optique.

Cet ouvrage propose successivement un rappel des donnes épidémiologiques sur le polype adénomateux et le cancer colonectal, une description détaillée des procédures pour la réalisation et linterpeétation de l'ecamen, et une description precise des signes permettant de poser le diagnostic de polype ou de masse colique, a la fois sur les images bidimensionnelles et sur les images tridimensionnelles.

Toutes les recettes pour déjouer les pickes de linterprétation y sont rapportées, y compris celles utilisunt des systèmes de détection automatique (CAD). Un chapitre est dédié à la problématique des lésions extracoliques detectées lors de lecamen. Un autre rapporte les indications actuclles de la coloscopie virnuellec, définies récemment pur la Haute Autorité de Santé.

Intéresse : tous les internes et chefs de clinique, etudiants en radiologie, radiologues, cancerologues, gastroentérologues.

En vente chez votre libraire spécialise, par correspondance ou sur notre site www medecine, lavoisier.fr ou wwwemintec.fr Bon de commande à retoumer complete a : LAVOISIER SAS - 14, rue de Provigny - 94236 CACHAN Cedex

Coloscopie virtuelle : 49 e TTC $\{+7$ e de participation $\quad$ Quantite aux frais de port par exemplaire) soit $56 \mathrm{e}$

frais de port offerts" a partir de $60 \mathrm{e}$ si paiement joint a la commande

* France metropolitaine, Suise, UE Autres, nous conwiter par mal t infoelavoisier for)

Carte bancaire $\mathrm{n}^{*}:$\begin{tabular}{|l|l|l|l|l|l|l|l|l|l|l|l|l|l|l|}
\hline & & & & & & & & & & & & & & \\
\hline
\end{tabular}

Les 3 dernien chiffres situés au dos de votre carte bancaire $1 \square^{\square} \mid \square$ Nom / Finom t....................................................

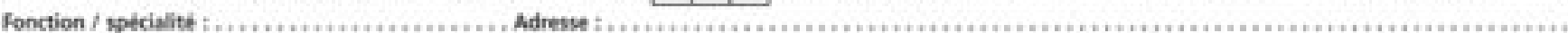

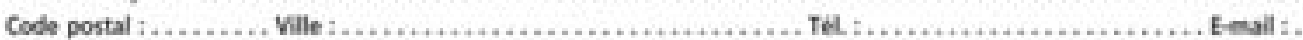

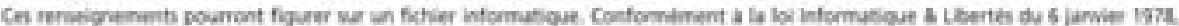

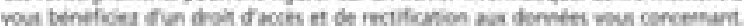

te joins mon règlement a la commande :

Montant total de :

Cheque bancaire ou postal payable en France a Forde de: LAVOISIER SAS (Une facture açuittée sera jointe au colis)

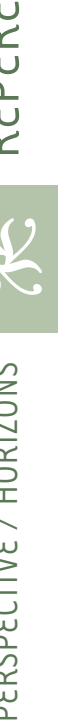



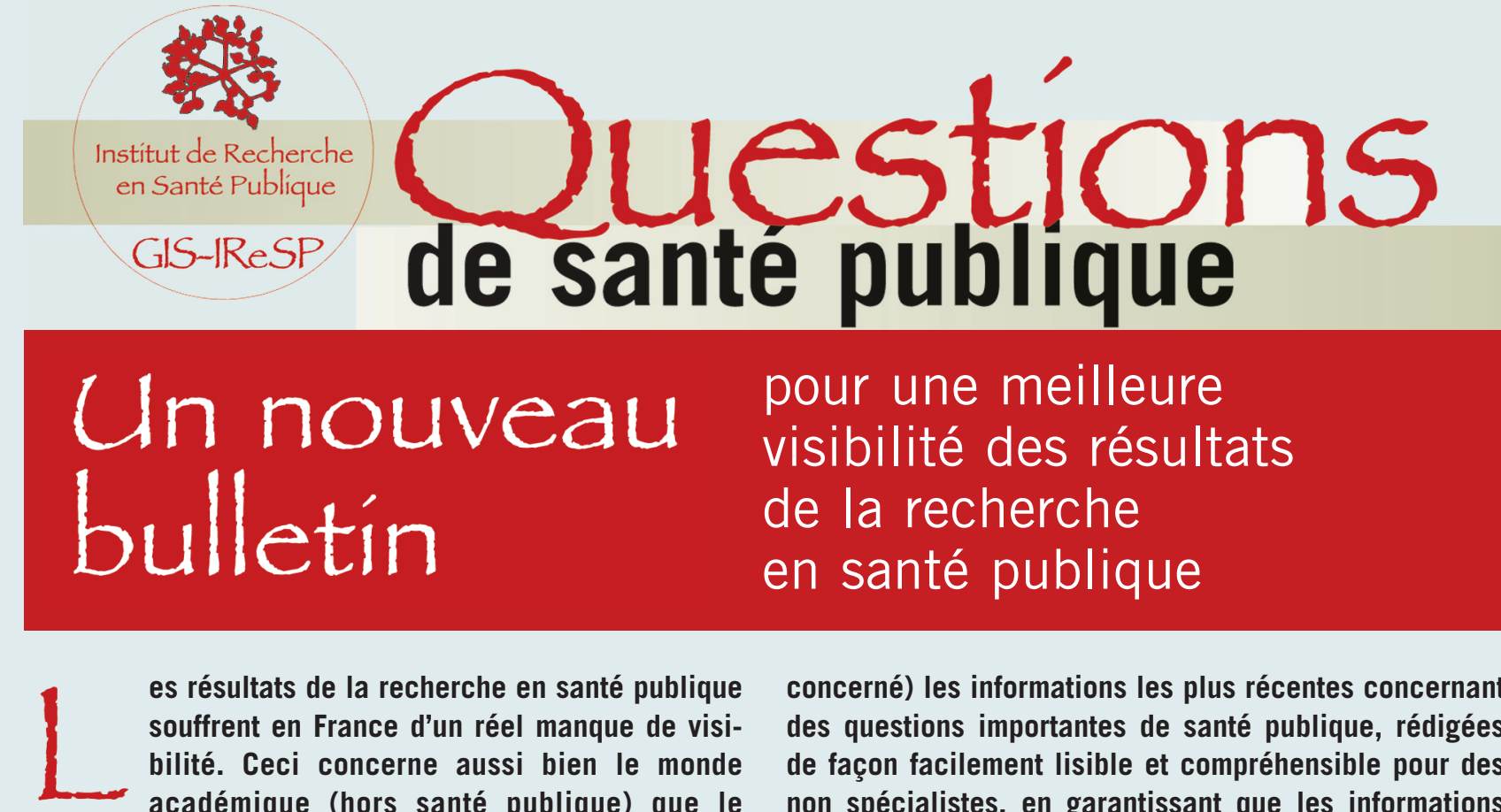

es résultats de la recherche en santé publique souffrent en France d'un réel manque de visibilité. Ceci concerne aussi bien le monde académique (hors santé publique) que le grand public et les décideurs. Pour pallier ce déficit, l'IReSP crée un bulletin à large diffusion intitulé " Questions de santé publique ", largement inspiré du bulletin mensuel d'information de I'INED «Populations et sociétés ". L'objectif éditorial est de porter à la connaissance d'un large public (enseignants, étudiants, journalistes, décideurs, milieux de la recherche, associations, public

concerné) les informations les plus récentes concernant des questions importantes de santé publique, rédigées de façon facilement lisible et compréhensible pour des non spécialistes, en garantissant que les informations publiées sont validées scientifiquement. La publications concernera des faits et non des positions. Au-delà de la présentation de résultats, cette publication devrait également avoir des qualités pédagogiques, permettant au lecteur de mieux comprendre comment sont formulées et abordées les questions de santé publique et quelles sont les limites de ces études.
Nom
Prénom
Institution
Fonction
Spécialité
Service
Adresse
Ville
Code postal
Pays
Adresse électronique
à nous retourner par la poste ou par fax au 0155641394
Questions de santé publique
Les Éditions EDK
2, rue Troyon
92310 Sèvres
France 